\title{
COMPLICAÇÕES BILIARES PÓS-TRANSPLANTE HEPÁTICO INTERVIVOS
}

\author{
BILIARY COMPLICATIONS AFTER LIVING DONOR LIVER TRANSPLANTATION
}

\author{
Júlio C. U. Coelho, TCBC-PR ${ }^{1}$; Jorge Eduardo Fouto Matias, TCBC-PR ${ }^{1}$; Giorgio Alfredo Pedroso Baretta'; \\ Adriane Celli ${ }^{1}$; Júlio Cesar Pisani² ${ }^{2}$ Jorge Massayukim Yokochi $^{3}$
}

\begin{abstract}
RESUMO: Objetivo: A reconstituição biliar no transplante hepático intervivos é associada à elevada taxa de complicações. O objetivo do presente estudo é apresentar a nossa experiência com as complicações biliares pós-transplante hepático intervivos e o seu tratamento. Método: De um total de 300 transplantes hepáticos, 51 (17\%) foram com doadores vivos. Todos receptores tinham o grupo sangǘńneo ABO idêntico aos dos doadores. Os prontuários eletrônicos dos receptores foram avaliados para determinar a presença e o tipo de anomalia da via biliar, o tipo de reconstituição da via biliar, presença de complicações vasculares e biliares e o método e o resultado do tratamento das complicações. Resultados: A via biliar era dupla em sete enxertos (16,7\%) e tripla em dois $(4,8 \%)$ enxertos do lobo hepático direito. Nos demais, ela era única. O tipo de reconstituição mais comum foi a hepaticohepaticostomia única ou dupla (38 transplantes; 75\%). Complicações biliares ocorreram em 21 pacientes $(41,2 \%)$ e incluíram fístula biliar em 11 $(21,6 \%)$, estenose biliar em seis $(11,8 \%)$ e fístula com estenose em quatro $(7,8 \%)$. O local da fístula foi na anastomose biliar em 11 pacientes $(21,6 \%)$ e na superfície cruenta do fígado em quatro $(7,8 \%)$. O tratamento consistiu de inserção de prótese biliar em oito, papilotomia em um, retransplante em dois que tinham trombose da artéria hepática e sutura do ducto em um. A fístula fechou com o tratamento conservador em três pacientes. A maioria dos pacientes com estenose biliar foi tratada com dilatação seguida da colocação de prótese biliar. Conclusões. As complicações biliares são freqüentes após o transplante hepático intervivos e são associadas à elevada taxa de morbidade e mortalidade (Rev. Col. Bras. Cir. 2005; 32(4): 195-200).
\end{abstract}

Descritores. Fístula biliar; Transplante de fígado; Complicações pós-operatórias.

\section{INTRODUÇÃO}

O transplante hepático intervivos (THI) foi realizado pela primeira vez em crianças por Raia et $_{\text {al }}{ }^{1}$ na Universidade de São Paulo em 1989 e em adultos por Tanaka et al ${ }^{2}$ na Universidade de Kyoto no Japão poucos anos após. Este procedimento foi conseqüente a uma série de avanços em técnica cirúrgica que possibilitou dividir o fígado em segmentos independentes e capazes de realizar adequadamente todas as funções do órgão. A secção precisa do parênquima hepático, vasos e vias biliares permite retirar alguns segmentos hepáticos de um doador vivo e implantá-los em um receptor pediátrico ou adulto.

A reconstituição biliar é considerada o tendão de Aquiles do transplante hepático porque as complicações biliares são freqüentes, aumentam a internação e o custo hospitalar, comprometem a qualidade de vida pós-transplante e geralmente necessitam tratamento intervencionista, como procedimentos percutâneos, endoscópicos e cirúrgicos ${ }^{3,4}$. A reconstituição biliar no THI é mais difícil e associada a maior incidência de complicações do que no transplante hepático cadavérico (THC) ${ }^{5}$. No THI, a anastomose biliar é realizada com o ducto hepático direito ou esquerdo e não com o colédoco como no $\mathrm{THC}^{6}$. Além do mais, variações anatômicas são freqüentes, com presença de dois ou mais ductos biliares no hilo dos lobos hepáticos, necessitando de mais de uma anastomose biliar com ductos de diâmetro bastante reduzi$\operatorname{dos}^{7,8}$. As complicações biliares pós-THI ainda não foram relatadas no nosso meio. O objetivo do presente estudo é apresentar as complicações biliares pós THI e o seu tratamento no nosso Serviço.

\section{MÉTODO}

De um total de 300 transplantes hepáticos realizados no Hospital de Clínicas da Universidade Federal do Paraná e no Hospital Nossa Senhora das Graças de Curitiba, pela mesma equipe médica, no período de 24 setembro de 1991 a 7 de agosto de 2004, 51 (17\%) foram transplantes com doadores vivos. O primeiro THI foi realizado em nosso Serviço em 28 de março de 1998.

As características demográficas dos receptores estão evidenciadas na Tabela 1. A idade dos receptores variou de 7 meses a 66 anos, com uma média de 43 anos. Trinta e quatro eram do sexo masculino $(66,7 \%)$ e 17 do feminino $(33,3 \%)$. Todos os receptores tinham o mesmo grupo sangüíneo $\mathrm{ABO}$ dos doadores.

A seleção do segmento hepático transplantado dependia da relação entre o peso do enxerto com o peso corporal do receptor, que era sempre maior do que 0,8 . O peso do enxerto era determinado pela volumetria do fígado e seus lobos através de ressonância magnética. A hepatectomia do

1. Membros do Serviço de Transplante Hepático do Hospital de Clínicas da Universidade Federal do Paraná, Curitiba

2. Professor Adjunto e Chefe do Serviço de Gastroenterologia do Hospital de Clínicas da Universidade Federal do Paraná, Curitiba

3. Radiologista do Hospital Vita de Curitiba

Recebido em 25//01/2005

Aceito para publicação em 16/06/2005

Conflito de interesse: nenhum

Fonte de financiamento: nenhuma

Trabalho realizado no Serviço de Transplante Hepático do Hospital de Clínicas da Universidade Federal do Paraná, Curitiba. 
Tabela 1 - Características Demográficas de 51 Receptores de Transplante Hepático Intervivos.

\begin{tabular}{ll}
\hline $\begin{array}{l}\text { Idade } \\
\quad \text { limites }\end{array}$ & 7 meses a 66 anos \\
$\quad$ média & 43 anos \\
Sexo & $34 \quad(66,7 \%)$ \\
$\quad$ masculino & $16(33,3 \%)$ \\
$\quad$ feminino & 51 idênticos \\
Compatibilidade ABO & \\
Enxerto & 42 \\
$\quad$ lobo direito & 1 \\
$\quad$ lobo esquerdo & \\
$\quad$ segmento lateral esquerdo & 8 \\
Seguimento & \\
$\quad$ limites & 1 dia a 6 anos e 10 meses \\
$\quad$ média & 1 ano e 5 meses \\
\hline
\end{tabular}

Tabela 2 - Indicação de Transplante Hepático Intervivos em 51 Pacientes.

\begin{tabular}{lrr}
\hline Diagnóstico & N & \% \\
\hline Cirrose VHC & 15 & 29,4 \\
Hepatite auto-imune & 6 & 11,8 \\
Atresia vias biliares & 6 & 11,8 \\
Cirrose VHB & 5 & 9,8 \\
Cirrose VHC + carcinoma hepatocelular & 4 & 7,8 \\
Cirrose VHB + carcinoma hepatocelular & 3 & 5,9 \\
Cirrose biliar primária & 3 & 5,9 \\
Cirrose alcoólica & 2 & 3,9 \\
Outros & 7 & 13,7 \\
\hline
\end{tabular}

$V H C=$ vírus da hepatite $C ; V H B=$ vírus da hepatite $B$

doador nunca excedia $65 \%$ do volume hepático total do doador. Todos os adultos receberam o lobo hepático direito (segmentos V a VIII) e as crianças, dependendo da relação do volume hepático do doador com o peso corpóreo do receptor, receberam o segmento lateral esquerdo (segmentos II e III) ou o lobo esquerdo (segmentos II, III e IV).

Com relação à classificação de Child-Pugh, dois $(3,9 \%)$ receptores eram Child A, 31 (60,8\%) Child B e 18 $(35,3 \%)$ Child C.

As indicações de transplante estão listadas na Tabela 2. As indicações mais comuns foram hepatopatia pelo vírus da hepatite $\mathrm{C}$ ou $\mathrm{B}$, hepatite auto-imune e atresia das vias biliares.

A técnica de transplante hepático intervivos foi descrita em outros artigos ${ }^{9-11}$.

A derivação veno-venosa não foi utilizada. As reconstruções biliares foram realizadas após a confecção das anastomoses vasculares. As anastomoses biliares foram feitas com fio monofilamentar absorvível de polidioxanona (PDS) 6-0 ou 7-0, com pontos separados. Os nós foram posicionados na face externa da anastomose, tanto na superfície posterior como anterior.

A reconstituição biliar foi realizada, sempre que possível, com anastomose término-terminal entre o ducto hepáti- co direito ou esquerdo do doador e o ducto hepático equivalente do receptor. Na presença de mais de um ducto biliar do doador, procedia-se, sempre que possível, à ductoplastia (secção do septo entre os ductos) para unificar os ductos e posteriormente, realizar uma única anastomose. Na impossibilidade de realizar ductoplastia, eram confeccionadas duas ou três anastomoses com o ducto biliar do receptor ou hepaticojejunostomia. Nos casos de possibilidade de tensão na anastomose e nos receptores com colangite esclerosante primária, a hepaticojejunostomia era o procedimento de escolha. Para a confecção desta anastomose, a primeira alça jejunal era seccionada e a sua bôca distal fechada com poligalactina 3-0, com sutura contínua em dois planos. A anastomose entre um ou mais ductos hepáticos e a alça distal foi realizada de maneira término-terminal com fio de polidioxanona. A alça proximal foi anastomosada de maneira término-terminal ao jejuno, a cerca de $40 \mathrm{~cm}$ da anastomose hepaticojejunal, com poligalactina 3-0, em dois planos.

Todas as anastomoses foram confeccionadas com o auxílio de lupa de magnificação de 3,5 de aumento. Drenagem biliar não foi utilizada em nenhum paciente. Dreno suctor duplo de grosso calibre foi colocado de rotina no espaço subfrênico e no hilo hepático. Todos os doadores e receptores eram submetidos a colangiorressonância no pré-operatório. Colangiografia per-operatória não foi realizada de rotina.

A imunossupressão utilizada consistia de tacrolimus $(n=42)$ ou ciclosporina $(n=7)$ com corticóide. Dois pacientes não receberam inibidores da calcineurina por apresentarem insuficiência renal no pós-operatório imediato e terem morrido precocemente.

Os prontuários eletrônicos contendo todos os dados dos receptores de THI foram avaliados retrospectivamente. Foram obtidos dados de anamnese, exame físico, exames complementares, anatomia das vias biliares e vasos hepáticos e os de reconstituição cirúrgica destas estruturas.

As variáveis analisadas incluíram: presença e o tipo de anomalia da via biliar, o tipo de reconstituição da via biliar, presença de complicações vasculares e biliares e o método e resultado do tratamento das complicações biliares. Considerou-se como anatomia normal da via biliar, quando o ducto hepático comum era formado pela união de um ducto biliar esquerdo com um direito no hilo hepático, com o ducto cístico sendo tributário do hepático comum ${ }^{12}$. O diagnóstico de complicação biliar foi estabelecido por dados clínicos, laboratoriais e de exames de imagem. O diagnóstico de trombose da artéria hepática foi estabelecido inicialmente por ultra-sonografia de Doppler e confirmado por arteriografia.

Este estudo foi aprovado pela Comissão de Ética do Hospital de Clínicas da Universidade Federal do Paraná (protocolo CEP/HC 759.178/2003-11).

\section{RESULTADOS}

A via biliar era única em todos os nove enxertos de segmento lateral esquerdo ou lobo hepático esquerdo e em 33 de 42 (78.6\%) dos de lobo hepático direito. A via biliar era dupla em sete $(16,7 \%)$ e tripla em dois $(4,8 \%)$ enxertos do lobo hepático direito. Os tipos de reconstituição biliar são 
Tabela 3 - Tipo de Reconstituição Biliar em 51 Transplantes Intervivos.

\begin{tabular}{lrr}
\hline Reconstrução & N & \% \\
\hline Hepaticohepaticostomia única & 30 & 58,8 \\
Ductoplastia + hepaticohepaticostomia única & 3 & 5,9 \\
Hepaticohepaticostomia dupla + ligadura 1 ducto & 2 & 3,9 \\
Hepaticohepaticostomia dupla & 2 & 3,9 \\
Hepaticohepaticostomia única + ligadura 1 ducto & 1 & 2,0 \\
Hepaticojejunostomia & 12 & 23,5 \\
Hepaticojejunostomia + hepaticohepaticostomia única & 1 & 2,0 \\
\hline
\end{tabular}

evidenciados na Tabela 3. O tipo de reconstituição mais comum foi a hepaticohepaticostomia única ou dupla (38 transplantes; $75 \%$ ). Ductoplastia foi realizada previamente à hepaticohepaticostomia em três receptores. Foi realizada hepaticohepaticostomia de somente um ducto biliar em um receptor que apresentava dois. O outro ducto de fino calibre foi ligado pela impossibilidade de realizar uma anastomose pérvia. Um receptor com dois ductos biliares foi submetido à hepaticohepaticostomia de um ducto e hepaticojejunostomia de outro ducto. Os dois enxertos com três ductos hepáticos direitos foram submetidos à hepaticohepaticostomia dupla $\mathrm{e}$ ligadura de um ducto de fino calibre.

Complicações biliares foram diagnosticadas em 21 pacientes $(41,2 \%)$ e incluíram fístula biliar em 11 pacientes $(21,6 \%)$, estenose biliar em seis $(11,8 \%)$ e fístula com estenose em quatro $(7,8 \%)$. A fístula biliar foi a complicação mais comum e ocorreu em 15 pacientes $(29,4 \%)$. O diagnóstico de fístula biliar foi estabelecido pela presença de bile no dreno suctor ou pela presença de coleção líquida intrabdominal identificada por ultra-sonografia ou tomografia, cuja drenagem percutânea confirmou a presença de bile. O local de extravasamento de bile foi identificado por colangiografia endoscópica retrógrada (oito casos), colangiografia transparietohepática (um caso), fistulografia pelo dreno suctor (um caso), colangiorressonância (um caso) ou durante laparotomia (um caso).

O diagnóstico de fístula biliar foi estabelecido entre o $4^{\circ}$ e o $60^{\circ}$ dia de pós- transplante, em uma média no $15^{\circ}$ dia. A Tabela 4 mostra o local, tratamento e evolução dos pacientes com fístula biliar. O extravasamento biliar ocorreu na anastomose biliar em 11 pacientes $(21,6 \%)$ e na superfície cruenta do fígado em quatro $(7,8 \%)$. Dos pacientes com fístula no local da anastomose biliar, 10 tinham hepaticohepaticostomia $(19,6 \%)$ e um hepaticojejunostomia (2\%). A fístula era de alto débito ( $>100 \mathrm{ml} /$ dia) em nove desses pacientes e de baixo $(<100$ $\mathrm{ml} / \mathrm{dia}$ ) em dois. O tratamento consistiu de inserção de prótese biliar por via endoscópica em sete pacientes e por via percutânea em um. Dos sete pacientes em que a prótese biliar endoscópica foi inserida, dois tiveram resolução, dois morreram e três tiveram fechamento da fístula somente após a conversão da hepaticohepaticostomia para hepaticojejunostomia. Dois desses pacientes tinham também estenose da anastomose biliar. Houve resolução da fístula no paciente em que a prótese biliar foi inserida por via percutânea. Dois pacientes, que apresentavam também trombose da artéria hepática, foram submetidos a re-transplante hepático cadavérico, sendo que um deles apresentou boa recuperação e outro morreu no $4^{\circ}$ dia de pós-operatório. Este último também apresentava estenose biliar. A fístula fechou apenas com papilotomia endoscópica em um paciente com fístula de baixo débito 48 dias após o transplante. Este paciente evoluiu posteriormente para estenose biliar.

Dos pacientes com fístula na superfície cruenta do fígado, o débito era baixo em três $(5,9 \%)$ e fechou com o tratamento conservador em um período de sete a 28 dias. $\mathrm{O} 4^{\circ}$ paciente, uma criança de quatro anos, apresentou ascite biliar e foi submetido a laparotomia no $19^{\circ}$ dia de pós-operatório. $\mathrm{Na}$ operação, foi identificado que o local de extravasamento de bile era um pequeno ducto biliar aberto na superfície cruenta do fígado. Este foi suturado e a criança apresentou boa recuperação, sem outras complicações.

Estenose biliar foi diagnosticada em 10 pacientes $(19,6 \%)$, sendo que seis tiveram somente estenose e quatro apresentaram inicialmente fístula e posteriormente estenose. O diagnóstico de estenose biliar foi suspeitado pela elevação de enzimas hepáticas, principalmente bilirrubinas, fosfatase alcalina e gamaglutamiltransferase e confirmado pela identificação de estenose na colangiorressonância ou colangiografia endoscópica ou percutânea.

O diagnóstico de estenose foi estabelecido do $1^{\circ}$ ao $12^{\circ}$ mês de pós-transplante, com uma média de 5,3 meses. A

Tabela 4 - Local, Tratamento e Evolução de Fístula Biliar em 15 Pacientes Submetidos a Transplante Hepático Intervivos.

\begin{tabular}{|c|c|c|}
\hline Local & Tratamento & Evolução \\
\hline \multirow[t]{4}{*}{ Anastomose biliar $(\mathrm{n}=11)$} & Prótese biliar endoscópica $(\mathrm{n}=7)$ & $\begin{array}{l}\text { Resolução após conversão da } \\
\text { anastomose biliodigestiva }(\mathrm{n}=3) \\
\text { Resolução }(\mathrm{n}=2) \\
\text { Óbito }(\mathrm{n}=2)\end{array}$ \\
\hline & Prótese biliar percutânea $(\mathrm{n}=1)$ & Resolução (n=1) \\
\hline & Transplante hepático $(\mathrm{n}=2)$ & $\begin{array}{l}\text { Resolução (n=1) } \\
\text { Óbito }(\mathrm{n}=1)\end{array}$ \\
\hline & Papilotomia $(\mathrm{n}=1)$ & Resolução (n=1) \\
\hline Superfície cruenta do fígado $(n=4)$ & $\begin{array}{l}\text { Conservador }(\mathrm{n}=3) \\
\text { Sutura ducto biliar }(\mathrm{n}=1)\end{array}$ & $\begin{array}{l}\text { Resoluçãa (n=3) } \\
\text { Resolução (n=1) }\end{array}$ \\
\hline
\end{tabular}


Tabela 5 - Tratamento e Evolução de Estenose Biliar em 10 Pacientes Submetidos a Transplante Hepático Intervivos.

\begin{tabular}{lll}
\hline Complicação & Tratamento & Evolução \\
\hline Somente Estenose $(\mathrm{n}=6)$ & $\begin{array}{l}\text { Dilatação + prótese biliar } \\
\text { endoscópica }(\mathrm{n}=5)\end{array}$ & $\begin{array}{l}\text { Resolução }(\mathrm{n}=3) \\
\text { Resolução após conversão da } \\
\text { anastomose biliodigestiva }(\mathrm{n}=1) \\
\text { Morte }(\mathrm{n}=1)\end{array}$ \\
& Dilatação + prótese biliar percutânea $(\mathrm{n}=1)$ & $\begin{array}{l}\text { Resolução }(\mathrm{n}=1) \\
\text { Estenose }+ \text { fístula }(\mathrm{n}=4)\end{array}$ \\
& $\begin{array}{l}\text { Resolução }(\mathrm{n}=2) \\
\text { biliodigestiva }(\mathrm{n}=2)\end{array}$ \\
& $\begin{array}{l}\text { Dilatação }+ \text { prótese biliar endoscópica }(\mathrm{n}=1) \\
\text { Re-transplante }(\mathrm{n}=1)\end{array}$ & $\begin{array}{l}\text { Resolução }(\mathrm{n}=1) \\
\text { Morte }(\mathrm{n}=1)\end{array}$ \\
& &
\end{tabular}

Tabela 5 evidencia o tratamento e evolução dos pacientes com estenose biliar. Dilatação seguida de colocação de prótese biliar por via endoscópica ou percutânea foi utilizada em todos pacientes que tinham somente estenose. $\mathrm{O}$ período de tratamento variou de três a seis meses, sendo necessárias uma a três sessões de tratamento. Dos seis pacientes que apresentavam somente estenose, quatro tiveram resolução da estenose, um resolução somente após conversão da hepaticohepaticostomia para hepaticojejunostomia e um morreu.

Dos quatro pacientes que tinham estenose e fístula biliar, dois tiveram resolução da complicação após conversão da hepaticohepaticostomia para hepaticojejunostomia, um teve resolução após dilatação e colocação de prótese biliar por via endoscópica e um morreu após re-transplante hepática.

A sobrevida dos pacientes em um ano foi de $71 \%$ e no período de seguimento do estudo (um ano e cinco meses) foi de $63 \%$. Quatro pacientes morreram de causas secundárias às complicações biliares: dois do grupo de fístula, um do grupo de estenose e um do grupo de fístula com estenose.

\section{DISCUSSÃO}

Complicações biliares são causas comuns de morbidade e mortalidade após transplante hepático ${ }^{13,14}$. A taxa elevada de complicações biliares é devida a vários fatores, incluindo isquemia das vias biliares, imunossupressão, transplante entre indivíduos ABO incompatíveis, infecção pelo citomegalovírus e fatores técnicos ${ }^{15,16}$. A vascularização arterial das vias biliares, inclusive do ducto hepático direito e esquerdo, é realizada quase que exclusivamente por vasos derivados da artéria hepática própria ${ }^{17,18}$. A lesão da artéria hepática ou de seus ramos pode causar isquemia das vias biliares. A dissecção mínima do ducto hepático direito ou esquerdo e a sua secção próxima ao hilo hepático durante o procedimento de hepatectomia no doador no THI são essenciais para evitar desvascularização das vias biliares ${ }^{3}$. Dissecção excessiva do ducto biliar compromete o seu suplimento sangüíneo e aumenta a incidência de complicações biliares ${ }^{3}$. A isquemia é uma importante causa de fístula e estenose biliar no transplante hepático. Trombose da artéria hepática aumenta não somente a incidência, mas principalmente a gravidade das complicações biliares. A maioria dos pacientes com trombose da artéria hepática necessita retransplante.

A imunossupressão também pode ser importante na patogênese das complicações biliares. Alguns imunossupressores inibem a resposta inflamatória e cicatricial normal necessária para o desenvolvimento de tecido fibrótico firme e maduro. Transplante entre indivíduos com tipo de sangue ABO incompatível ou infecção causada pelo citomegalovírus também aumenta a incidência de complicações biliares ${ }^{5}$. O principal mecanismo de lesões provocadas pelo citomegalovírus é uma vasculite viral.

Fatores técnicos são de extrema importância na patogênese das complicações biliares pós-transplante hepático, principalmente nos THI. Neste tipo de transplante, a reconstrução biliar é realizada com o ducto hepático direito ou esquerdo, ducto de pequeno calibre, junto ao parênquima hepático. A dificuldade é ainda maior quando o doador apresenta dois ou três ductos biliares no lobo direito que se unem muito próximo da junção do ducto hepático direito com o esquerdo. Nesta situação, a secção do ducto hepático direito pode produzir dois ou três ductos biliares diminutos, que necessitam ser reconstruídos através de ductoplastia ou de dois ou três anastomoses biliares ${ }^{19,20}$. Além do mais, anomalias congênitas em que ductos biliares acessórios drenam no ducto hepático do lado oposto ou no ducto hepático comum são observadas em alguns indivíduos. Estas variações freqüentemente também necessitam de reconstrução apropriada para o sucesso do transplante. Apesar de existir relação entre o calibre e o número de ductos e a ocorrência de fístula e ou estenose biliar ${ }^{5-8}$, este dado não pode ser avaliado no nosso estudo pelo número limitado de pacientes.

As variações anatômicas são mais comuns no lobo hepático direito do que no esquerdo. O ducto hepático esquerdo é mais longo e em mais de $90 \%$ dos enxertos do lobo esquerdo ou dos segmentos laterais esquerdos, existe somente uma via biliar única para reconstrução ${ }^{18,21,22}$. A via biliar direita sofre ramificação precoce e é sujeita a um número maior de variações anatômicas. Ducto biliar direito único tem sido observado em 54 a $81 \%$ dos $\operatorname{casos}^{5,6,23}$. Em nosso estudo, via biliar única foi observada em todos enxertos do segmento lateral esquerdo ou lobo hepático esquerdo. Entretanto, via biliar única foi identificada em $78,6 \%$ dos enxertos do lobo 
hepático direito. O reconhecimento de mais de um ducto biliar e a sua adequada reconstrução é essencial para evitar atrofia ou infecção do segmento hepático correspondente ${ }^{5}$. Se a distância entre os orifícios dos ductos for pequena, estes podem ser unidos por meio de ductoplastia, de modo a permitir a realização de somente uma anastomose ${ }^{6}$. Esta técnica foi realizada em três dos nossos pacientes.

A reconstrução biliar pode ser feita com a via biliar do receptor ou com uma alça jejunal em Y de Roux, sendo que a maioria dos autores prefere a primeira técnica ${ }^{6,15,24}$. No caso de dois ductos biliares, pode-se anastomosar um ducto com o hepático direito e o outro com o hepático esquerdo ou mesmo com o ducto cístico. No caso de três ductos, pode-se empregar está técnica (hepaticohepaticostomia dupla), com ligadura de um dos ductos, se este for de fino calibre, o que impossibilitaria uma anastomose pervia. Caso contrário, deve-se realizar hepaticojejunostomia tripla.

As taxas de complicações biliares variam de 10 a $64 \%$ após THI e são mais elevadas do que as taxas de cinco a $30 \%$ observadas após transplante hepático cadavérico ${ }^{5,6,13,24}$. A incidência de complicações biliares em nossa experiência foi de $41 \%$, muito superior à taxa de $18 \%$ relatada pelo nosso grupo após 147 transplantes hepáticos cadavéricos ${ }^{16}$. A experiência da equipe de cirurgia parece ser fundamental para reduzir a incidência dessas complicações.Fan et $a l^{6}$ relataram incidência de $43 \%$ de complicações biliares nos primeiros 37 pacientes submetidos a THI e de somente $8 \%$ nos 37 subseqüentes.

A apresentação clínica das fístulas biliares é mais precoce do que a das estenoses ${ }^{5}$. Na presente casuística, as fístulas foram diagnosticadas em média duas semanas após o transplante, enquanto que as estenoses em cinco meses. Além do mais, algumas estenoses são secundárias à cicatrização de fístulas ou revisão cirúrgica das mesmas ${ }^{24}$.

Com os avanços tecnológicos dos últimos anos, o tratamento das complicações biliares deixou de ser unicamente cirúrgico e passou a ser multidisciplinar ${ }^{25-27}$. Na presença de fístula biliar, a complicação mais freqüente pós-THI, a conduta inicial é estabelecer uma drenagem biliar externa ou interna adequada, de modo a evitar acúmulo de bile intrabdominal e as suas conseqüências, como inapetência, náuseas, vômitos, febre e mesmo sepse.
Este tratamento é suficiente para a maioria dos pacientes com fístula biliar na superfície cruenta do fígado. Raramente, como em um dos nossos pacientes, é necessária a sutura do ducto biliar da superfície hepática. Entretanto, a colocação de prótese biliar por via endoscópica ou percutânea é necessária para facilitar a drenagem biliar interna e assim reduzir o débito de bile pela fístula e reduzir o tempo de cicatrização no local de drenagem na anastomose. Somente nos pacientes que não apresentam resolução com esta conduta, está indicada revisão da anastomose, geralmente convertendo a hepaticohepaticostomia para hepaticojejunostomia em Y de Roux. Na nossa experiência, a maioria das fístulas da anastomose pós-THI resolveram com o tratamento endoscópico ou percutâneo. Park et al ${ }^{25}$ colaboradores relataram resolução de $100 \%$ dessas fístulas com o tratamento endoscópico e $78 \%$ com o tratamento percutâneo.

A maioria dos pacientes com estenose biliar pós-THI é eficazmente tratada com dilatação com balão seguida de colocação de prótese biliar por via endoscópica ou percutânea ${ }^{25}$. Muitos pacientes com fístula biliar desenvolvem posteriormente ou apresentam simultaneamente estenose biliar ${ }^{24}$. Nos casos de persistência ou recidiva da estenose após dilatação, está indicado o tratamento cirúrgico, com conversão da hepaticohepaticostomia para hepaticojejunostomia. Se o paciente já tiver uma hepaticojejunostomia, está indicada a revisão da derivação. A presença de dreno biliar inserido por via endoscópica ou percutânea, facilita a identificação da via biliar, reduzindo a possibilidade de lesão arterial do enxerto. A ultrasonografia per-operatória também pode ser útil para localizar o ducto biliar e a artéria hepática ${ }^{6}$.

Os pacientes, principalmente os adultos, com fístula e ou estenose biliar secundária a trombose da artéria hepática quase sempre necessitam re-transplante hepático ${ }^{28}$. Os procedimentos endoscópicos ou percutâneos são freqüentemente utilizados para amenizar as manifestações clínicas e as complicações, enquanto o paciente aguarda o re-transplante. A mortalidade do re-transplante nestes pacientes é elevada. Dos dois pacientes da nossa série que foram re-transplantados por trombose da artéria hepática, um morreu. Conclui-se deste estudo que as complicações biliares são freqüentes após o THI e são associadas a elevada taxa de morbidade e mortalidade.

\begin{abstract}
Background: Biliary reconstruction in living donor liver transplantation is associated with an elevated rate of complications. The objective of the present study is to present our experience with biliary complications following living donor liver transplantation and their treatment. Methods: Of a total of 300 hepatic transplantations, 51 (17\%) were of living donors. All receptors had identical ABO blood group of the donors. Electronic charts of the receptors were evaluated to determine the presence and type of biliary tree anomalies, presence of vascular and biliary complications and technique and results of the treatment of the complications. Results: The biliary duct was double in 7 grafts (16.7\%) and triple in $2(4.8 \%)$ grafts of the right lobe. In the remaining, the duct was single. The most common reconstitution procedure was single or double hepaticohepaticostomy $(n=38 ; 75 \%)$. Biliary complications occurred in 21 patients $(41.2 \%)$ and included biliary fistula in 11 (21.6\%), biliary stenosis in $6(11.8 \%)$, and fistula with stenosis in 4 (7.8\%). The fistula was at the biliary anastomosis in 11 patients (21.6\%) and at the liver surface in $4(7.8 \%)$. Treatment consisted of biliary stent insertion in 8, papillotomy in 1, retransplantation in 2 who had hepatic artery thrombosis, and suture of the biliary duct in 1. Fistula closed with conservative treatment in 3 patients. Most biliary stenosis was treated with dilation followed by biliary stent insertion. Conclusions: Biliary complications are frequent after living donor liver transplantation and they are associated with elevated rates of morbidity and mortality.
\end{abstract}

Key words: Biliary fistula; Liver transplantation; Postoperative complications. 


\section{REFERÊNCIAS}

1. Raia S, Nery JR, Mies S. Liver transplantation from live donors. Lancet. 1989; 2(8661):497.

2. Tanaka K, Uemoto S, Tokunaga Y, et al. Surgical techniques and innovations in living related liver transplantation. Ann Surg. 1993; 217(1):82-91.

3. Kawachi S, Shimazu M, Wakabayashi G, et al. Biliary complications in adult living donor liver transplantation with duct-to-duct hepaticocholedochostomy or Roux-en-Y hepaticojejunostomy biliary reconstruction. Surgery. 2002; 132(1):48-56.

4. Sutcliffe R, Maguire D, Mróz A, et al. Bile duct strictures after adult liver transplantation: a role for biliary reconstructive surgery? Liver Transplant. 2004; 10(7):928-34.

5. Settmacher U, Steinnmuller TH, Schmidt SC, et al. Technique of bile duct reconstruction and management of biliary complications in right lobe living donor liver transplantation. Clin Transplant. 2003; 17(1):37-42.

6. Fan ST, Lo CM, Liu CL, et al. Biliary reconstruction and complications of right lobe live-donor liver transplantation. Ann Surg. 2002; 236(5):676-83.

7. Liu CL, Lo CM, Chan SC, et al. Safety of duct-to-duct biliary reconstruction in right lobe live-donor liver transplantation without biliary drainage. Transplantation. 2004; 77(5):726-32.

8. Ishiko T, Egawa H, Kasahara M, et al. Duct-to-duct reconstruction in living donor liver transplantation utilizing right lobe graft. Ann Surg. 2002; 236(2):235-40.

9. Carone E, Chapchap P, Porta G, et al. Transplante hepático com doador vivo familiar. J Pediatr. 1998; 74(2):99-106.

10. Carone E, Chapchap P, Pugliese V, et al. Transplante hepático com doador vivo familiar: técnica operatória no doador. Rev Col Bras Cir. 1997; 24(4):235-41.

11. Coelho JCU. Transplante hepático intervivos. In: Habr-Gama A, Gama-Rodrigues J, Machado MCC, et al, editores. Atualização em cirurgia do aparelho digestivo e coloproctologia. São Paulo: Frôntis Editorial; 2002. p. 159-64.

12. Testut L, Latarjet A. Tratado de Anatomia Humana. Barcelona: Salvat; 1986.

13. Miller CM, Gondolesi GE, Florman S, et al. One hundred nine living donor liver transplants in adults and children: a singlecenter experience. Ann Surg. 2001; 4(3):301-12.

14. Broelsch CE, Frilling A, Testa G, et al. Early and late complications in the recipient of an adult living donor liver. Liver Transplant. 2003; 9(10):S50-S53.

15. Kiuchi T, Ishiko T, Nakamura T, et al. Duct-to-duct biliary reconstruction in living donor liver transplantation. Transpl Proc. 2001; 33(3):1320-1.
16. Freitas ACT, Coelho JCU, Parolin MB, et al. Fatores de risco e conduta nas complicações do trato biliar no transplante hepático. Rev Col Bras Cir. 2000; 27(6):359-65.

17. Stapleton GN, Hickman R, Terblanche J. Blood supply of the right and left hepatic ducts. Br J Surg. 1998; 85(2):202-7.

18. Renz JF, Reichert PR, Emond JC. Biliary anatomy as applied to pediatric living donor and split-liver transplantation. Liver Transplant. 2000; 6(6):801-4.

19. Ho MC, Wu YM, Hu RH, et al. Surgical complications and outcome of living related liver transplantation. Transpl Proc. 2004; 36(8):2249-51.

20. Dulundu E, Sugawara Y, Sano K, et al. Duct-to-duct biliary reconstruction in adult living-donor liver transplantation. Transplantation. 2004; 78(4):574-9

21. Tannuri U, Velhote MC, Santos MM, et al. Pediatric liver transplantation: fourteen years of experience at the Children Institute in Sao Paulo, Brazil. Transplant Proc.2004; 36(4):941-2.

22. Gondolesi GE, Varotti G, Florman SS, et al. Biliary complications in 96 consecutive right lobe living donor transplant recipients. Transplantation. 2004; 77(12):1842-8.

23. Yoshida J, Chijiwa K, Yamaguchi K, et al. Practical classification of the branching types of the biliary tree: an analysis of 1094 consecutive direct cholangiograms. J Am Coll Surg. 1996; 182(3):37-40.

24. Testa G, Malago M, Valentin-Gamazo C, et al. Biliary anastomosis in living related liver transplantation using the right lobe: technique and complications. Liver Transplant. 2000; 6(6):710-14.

25. Park JS, Kim M-H, Lee SK, et al. Efficacy of endoscopic and percutaneous treatments for biliary complications after cadaveric and living donor liver transplantation. Gastrointest Endosc. 2003; 57(1):78-85.

26. Morelli J, Mulcahy HE, Willner IR, et al. Long-term outcomes for patients with post-liver transplant anastomotic biliary strictures treated by endoscopic stent placement. Gastrointest Endosc. 2003; 58(3):374-9.

27. Shar JN, Ahmad NA, Shetty K, et al. Endoscopic management of biliary complications after adult living donor liver transplantation. Am J Gastroenterol. 2004; 99(7):1291-5.

28. Kling K, Lau H, Colombani P. Biliary complications of living related pediatric liver transplant patients. Pediatr Transplant. 2004; 8(2):178-84.

Endereço para correspondência:

Dr. Júlio Coelho

Rua Bento Viana, 1140 - Ap.2202

80240-110 - Curitiba (PR)

Fone/Fax: (41) 322-3789 